\title{
A-5. A New Radio-Stereography in the Neurological Surgery
}

\author{
-On the Cerebral Angiography with a Lenticular Sheet- \\ Suyong Kwak, Yasuichi Ueda and Keiji Sano \\ Department of Neurological Surgery, Faculty of Medicine, University of Tokyo
}

It has been well accepted that three dimensional roentgenograms could give more information in the neurological surgery. However viewing of these pictures has a lot of disadvantages in the way of reproducing stereoscopic images, the techniques in making stereoscopic pictures are simple.

We applied a 'Lenticular Sheet' for viewing stereoscopic films on about 150 cases. Through a lenticular sheet as illustrated figures, one can separate images underneath it and create stereoscopic pairs from a picture. The principle of this kinds of 'Integral Photography' was described by G. Lippmann in 1908. With a lenticular stereogram one can observe the three dimensional image without any access of devices.

This method could be characterized since it is;
a) easy to handle,
b) useful to educate,
c) possible to print,
d) possible to preserve,
e) possible to exhibit.

This technique could be applied not only stereoscopic x-ray film but also stereo-

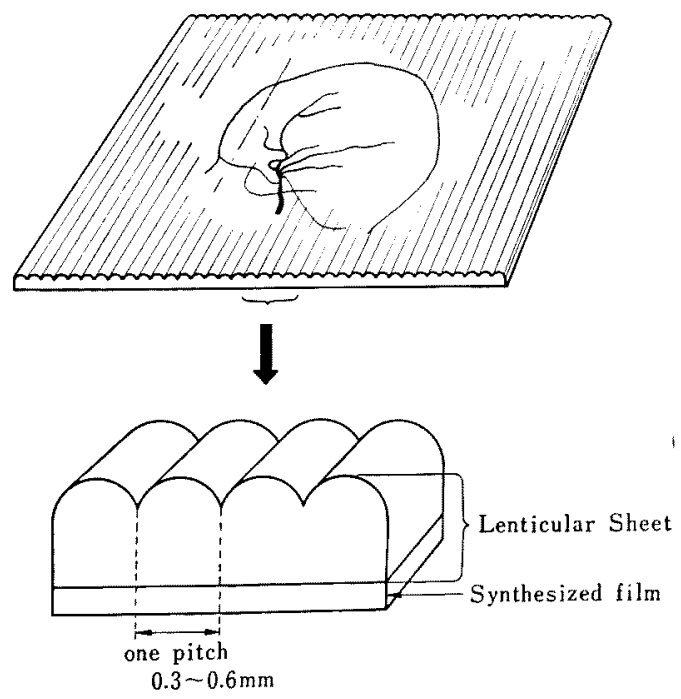

Fig. 1 Model Picture

$$
-213-
$$




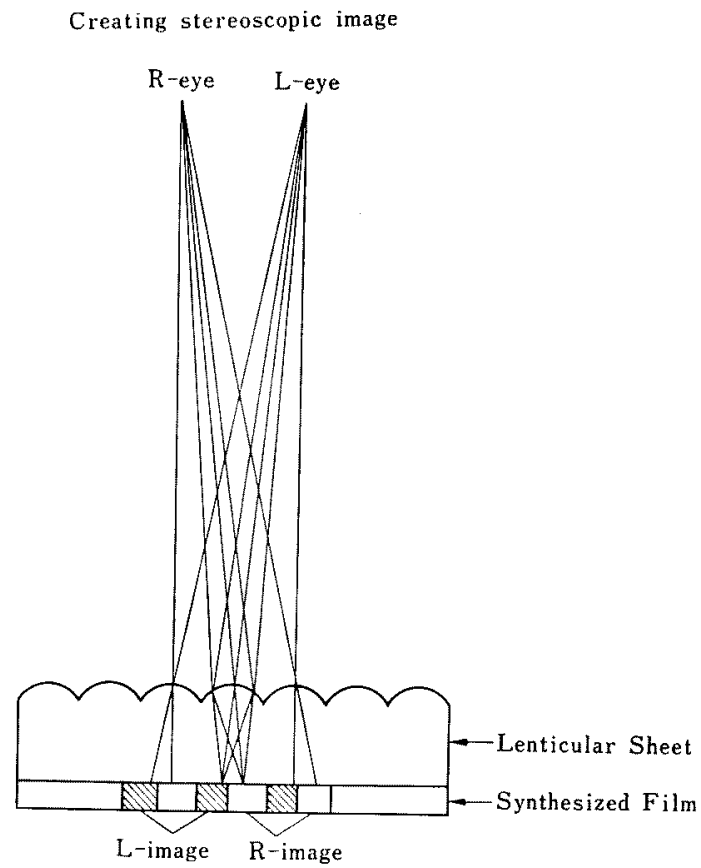

Fig. 2 Optical principle of separation of images by Lenticular Sheet.

scopic photography, stereoscopic projection and stereoscopic television and cinematography.

One can correlate the findings at the time of craniotomy without any devices.

The magnification in details is photographically possible.

If the four films which are combined of a pair of arterial phase and another pair of venous phase are combined, with an aid of each pair in different colors, stereoscopic whole vessel study can be achieved from a single plate. With this picture one can be able to have more exact geometry of the brain.

A practical measurement in depth will be discussed in the other paper.

\section{A-6. Microstereoangiography}

\section{Takayoshi Matusi and Keiji SANo} Keiichi Amano

Department of Neurosurgery, University of Tokyo

Department of Neurosurgery, Montreal Neurological Institute and McGill University, Montreal, Canada

Recent advance in roentgen-stereography has clarified definite importance of precise preoperative assessment of stereoscopic X-ray films toward diagnosis and 